\title{
P05.41. Development and evaluation of group medical visits for medically underserved women with chronic pelvic pain
}

\author{
M Chao ${ }^{*}$ P Abercrombie \\ From International Research Congress on Integrative Medicine and Health 2012 \\ Portland, Oregon, USA. 15-18 May 2012
}

\section{Purpose}

Chronic pelvic pain (CPP) severely impacts quality of life and is one of the most common medical problems among women. Effective management of CPP is needed, particularly for underserved women with limited access to quality care. We developed and evaluated group medical visits for women with CPP drawing from: (1) the Centering model, an innovative approach to groupbased healthcare across the lifecourse; and (2) integrative medicine emphasizing quality of life through patient-centered care.

\section{Methods}

Curriculum for ten monthly group visits of CenteringIMPPACT (Centering and Integrative Medicine for chronic Pelvic PAin Comprehensive Treatment) included mind-body approaches to managing CPP. A pilot study was conducted among patients with CPP at a safety net hospital. Qualitative and quantitative data were collected at baseline and three follow-up time points to assess feasibility, acceptability, and preliminary data on efficacy.

\section{Results}

CenteringIMPPACT participants $(n=12)$ were on average 42 years of age; $58 \%$ were African American; $42 \%$ had attended some college; and $83 \%$ had household incomes of less than $\$ 35,000 ; 42 \%$ reported being in fair or poor health. From baseline to 3-month follow-up, average number of days that women reported limitations of usual activities decreased from 24 to 15 . Health- related quality of life improved slightly; average life interference due to CPP decreased from 2.64 to 2.43. Mental health did not improve: scores on the Patient Health Questionnaire PHQ-9 for Depression increased from 11.25 to 12.43 . Based on qualitative data, participants experienced healthcare empowerment through learning to advocate for themselves and increased support and hope from the groups.

\section{Conclusion}

Preliminary data from this study indicates some improvements in functional activities and health-related quality of life among medically underserved women with CPP. Group medical visits are a viable model of comprehensive care for CPP in safety net healthcare settings. Additional data are needed to further evaluate the effects of CenteringIMPPACT.

Published: 12 June 2012

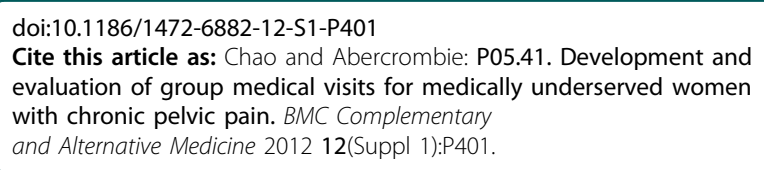

Cite this article as: Chao and Abercrombie: P05.41. Development and evaluation of group medical visits for medically underserved women with chronic pelvic pain. BMC Complementary and Alternative Medicine 2012 12(Suppl 1):P401.

Univ of California, San Francisco, Osher Center for Integrative Med, San Francisco, USA
() 2012 Chao and Abercrombie; licensee BioMed Central Ltd. This is an Open Access article distributed under the terms of the Creative Commons Attribution License (http://creativecommons.org/licenses/by/2.0), which permits unrestricted use, distribution, and reproduction in any medium, provided the original work is properly cited. 